\title{
The Effect of Nonuniform Pixel Responses in CCD on Quantitative Analysis
}

Binbin Wang ${ }^{1,2}$, Bryan D. Esser ${ }^{1}$, Nuria Bagués ${ }^{1}$, Jiaqiang Yan $^{3}$, Ari N. Blumer ${ }^{1,2}$ and David W. $\mathrm{McComb}^{1,2 *}$

1. Center for Electron Microscopy and Analysis, The Ohio State University, Columbus, OH USA.

2. Dept. of Materials Science \& Engineering, The Ohio State University, Columbus, OH, USA

3. Materials Science and Technology Division, Oak Ridge National Laboratory, TN 37831, USA

* Corresponding author: mccomb.29@osu.edu

Convergent beam electron diffraction (CBED) is a well-established technique that can give local information about a specimen, including structure [1], polarization [2] and charge density [3]. Improvements in electron optics such spherical aberration correction has enabled the formation of subangstrom electron probes. When a sub-angstrom probe is scanned across the specimen it can be observed that the diffraction information varies as the probe samples different positions within a unit cell. To get equivalent diffraction space information as conventional CBED, diffraction patterns are incoherently summed across a selected region of specimen larger than the unit cell, called position-averaged convergent beam electron diffraction (PACBED). Due to the rich phase information in CBED/PACBED, it has been shown an effective approach to quantitatively measure local properties like thickness [4], octahedral rotations [5], strain [6], and composition [7] in a wide range of materials.

In order to interpret and quantify the results of CBED/PACBED experiments, detailed comparison of the experimental data with simulations based well-characterized experimental conditions is critical [8]. In most cases, CBED/PACBED patterns are collected on charge coupled device (CCD) cameras, which can suffer from non-linear response functions across the detector [9]. While CCDs typically have routine gain normalization routines to account for such inconsistencies, improper application of these gain correction routines can adversely affect the quantitative interpretation of diffraction data in rather subtle ways. Therefore, care must be taken in quantitative interpretation of images from CCDs, particularly due to the nonuniform gain (flat field) and nonlinear response effects of such detectors. In this work, we demonstrate the negative effect of such non-linear gain across a CCD. In addition, we present one possible method for improving quantitative interpretation in the presence of residual non-linear gain.

As shown in Fig.1, PACBED of $\mathrm{LaFeO}_{3}$ near the [010] zone axis was acquired using the upper left quadrant of the Gatan UltraScan CCD in a probe aberration-corrected FEI Titan at $300 \mathrm{kV}$ with 2 seconds integration time and $10.2 \mathrm{mrad}$ convergence semi-angle. While the gain normalization of the camera was acceptable for most applications, there was measurable differences between the quadrants, thus making it advantageous to use just one quadrant. As indicated in Fig.1a, the pattern is slightly off zone in the [110] direction; however, the background contrast, as measured at the corners of the CCD (yellow boxes), indicates a residual non-uniformity in the gain normalization, as shown in Fig. 1 b.

A series of structures with different octahedral tilts is used to simulate the PACBED of $\mathrm{LaFeO}_{3}$ along [010] beam direction using the quantum excitation of phonons model [10]. In Fig.2, $\chi^{2}$ maps quantifying the difference between simulated and experimental PACBED is to show their structural agreement. Thus, the minimum intensity in each map indicates the best agreement with experiment. In Fig.2a-c, different cutoffs are applied to reduce the effects of nonuniform gain. With the increases of the background cutoffs, the best agreement in each figure shows a trend to come near the expected tilts value. Nevertheless, the best agreement in Fig.2a still has a discrepancy with the expected $\alpha$ tilt, which is largely parallel to beam direction, as reported by Hwang et al [5]. 
References:

[1] A Genoni et al., Chemistry-A European Journal 24 (2018), p. 10881.

[2] J Taftø and J Spence, Journal of Applied Crystallography 15 (1982), p. 60.

[3] N Shibata et al., Nature communications 8 (2017), p. 15631.

[4] JM LeBeau et al., Ultramicroscopy 110 (2010), p. 118.

[5] J Hwang et al., Physical Review B 87 (2013), p. 060101.

[6] R Pantel et al., Microscopy and Microanalysis 9 (2003), p. 866.

[7] I MacLaren et al., Microscopy and Microanalysis 24 (2018), p. 180.

[8] J Hwang et al., Applied Physics Letters 100 (2012), p.

[9] TC Williams and CR Shaddix, Review of Scientific Instruments 78 (2007), p. 123702.

[10] LJ Allen and S Findlay, Ultramicroscopy 151 (2015), p. 11.

[11] SM Selbach et al., Journal of Solid State Chemistry 196 (2012), p. 249.

[12] Funding for this research was provided by the Center for Emergent Materials at the Ohio State University, an NSF MRSEC (Award Number DMR-1420451).

(a)

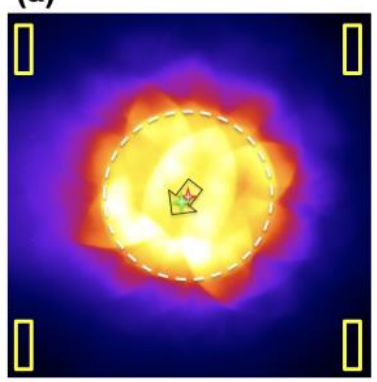

(b)

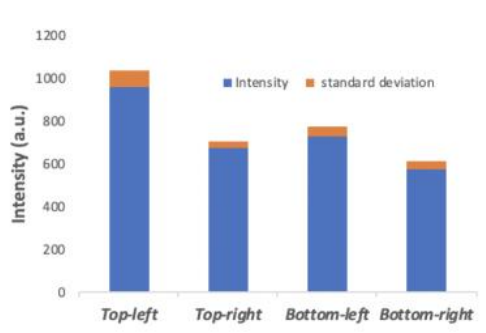

Figure 1. (a) $\mathrm{PACBED}$ of $\mathrm{LaFeO}_{3}$ near [010]. The arrow indicates the tilt direction while the cross marks are the center of mass (green) and center of the transmitted disk (red). (b) Background intensity variation within boxes as indicated in (a).

(a)

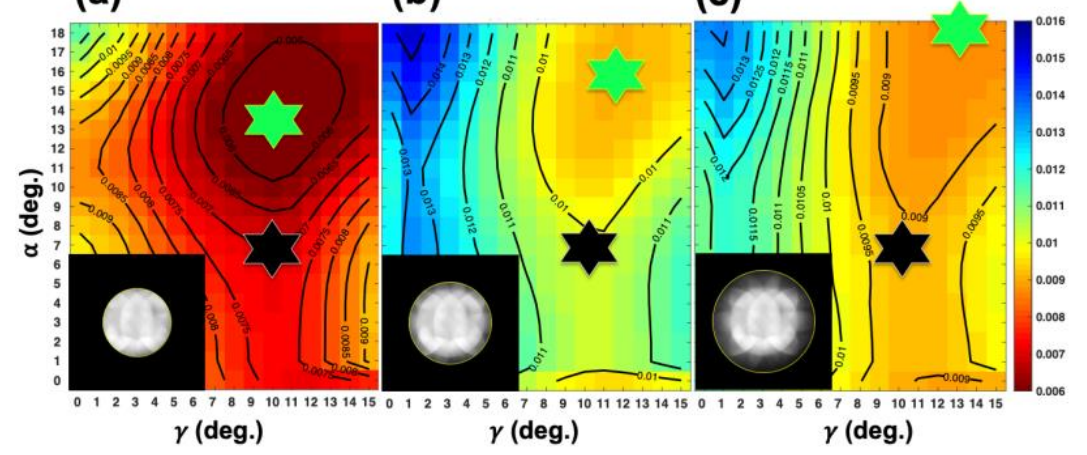

Figure 2. Quantification of the octahedral tilts in $\mathrm{LaFeO}_{3}$ by measuring the agreement between experimental PACBED and simulation using a $\chi^{2}$ function (color scale is normalized). Detector nonuniformity strongly affects the results as shown by applying various cutoff angles of (a) $1 \alpha$, (b) $1.2 \alpha$, (a) $1.5 \alpha$ to the experimental data. The stars drawn in each figure are the best agreement tilts (green) and the expected tilts (black) according [11]. 